\title{
EXTRAGALACTIC OBJECTS: AN EXAMPLE OF POPULARIZING ASTRONOMY FOR THE AMATEUR ASTRONOMER
}

\section{J.V. Feitzinger}

Observatory of the City of Bochum and Astronomical Institute, Ruhr-University, Postfach 10 2148, D-4630 Bochum 1, Federal Republic of Germany

\section{Introduction}

The handbook Astronomy (Handbuch für Sternfreunde), edited by G.D. Roth, will soon appear in its fourth edition. Newly written by 25 authors, the two volumes contain an additional chapter: Extragalactic Objects. The intention of my 50-page contribution to these volumes is to show the amateur astronomer that extragalactic work is not disappointing, ending in the northern sky with a fuzzy picture of the Andromeda Nebula.

The former editions of Roth's handbook were mainly characterized by the statement that the observation of extragalactic objects is beyond the capabilities of the amateur. In the last ten years the situation has drastically changed, especially because of new photographic techniques and sensitive emulsions and the availability of CCD's even for amateur astronomers. Small telescopes are no hindrance to doing interesting extragalactic work.

\section{Simplification}

To lower the threshold fear for the beginner, illustrative galaxy pictures are used. The pictures were taken by many different telescopes and cameras. Active German amateur astronomers contributed exclusively to this picture gallery.

A further way to lower the threshold fear follows the path of scientific research. In simple experimental steps, the amateur should learn how successful observations are done.

For example:

\begin{tabular}{ll|} 
Observing $\longrightarrow \begin{array}{l}\text { inventing concepts } \\
\text { making experiments } \\
\text { controlling variables }\end{array}$ & photography, processing \\
Classifying $\longrightarrow$ & $\begin{array}{l}\text { questioning } \\
\text { interpreting data } \\
\text { galaxies }\end{array} \mid$
\end{tabular}

Since the level of a broad, mixed educated readership has to be met, simplifica- 
tion can be used in a ninefold manner: 1) restrict to qualitative statements 2) idealize 3 ) simplify the statement 4) simplify the reasons for the statement 5) simplify by omission 6) generalize 7) particularize 8) use models with some special components 9 ) go back to the historic evolution; this offers often a simplified, naive picture.

Working with "simplification", the permissible level has to be controlled. The three controls are: 1) the simplification must be appropriate to the mixed levels of education of the readership 2) the simplification must continue and not block further, more difficult steps 3 ) the simplification must be technically relevant. Technical relevance and false information must always be distinguished. Technical relevance is most important.

\section{Organization and Examples}

The approach - "extragalactic objects" — is subdivided into the following sections: 1) catalogues and picture material 2) classification of galaxies 3) interacting galaxies and galaxies with peculiarities 4) structure of galaxies 5) general characteristics 6) brightness and color 7) stars and gas 8) mass and luminosity 9) structure formation in galaxies 10) spiral structure 11) cosmic cycles and energy distribution 12) distances 13) active galaxies and quasars 14) the universe 15) amateur techniques and amateur tasks. The emphasis of the different sections is: Sections 1-3: "find and look"; 4-8: "gather facts and think"; 9-14: "understand"; and 15: "work."

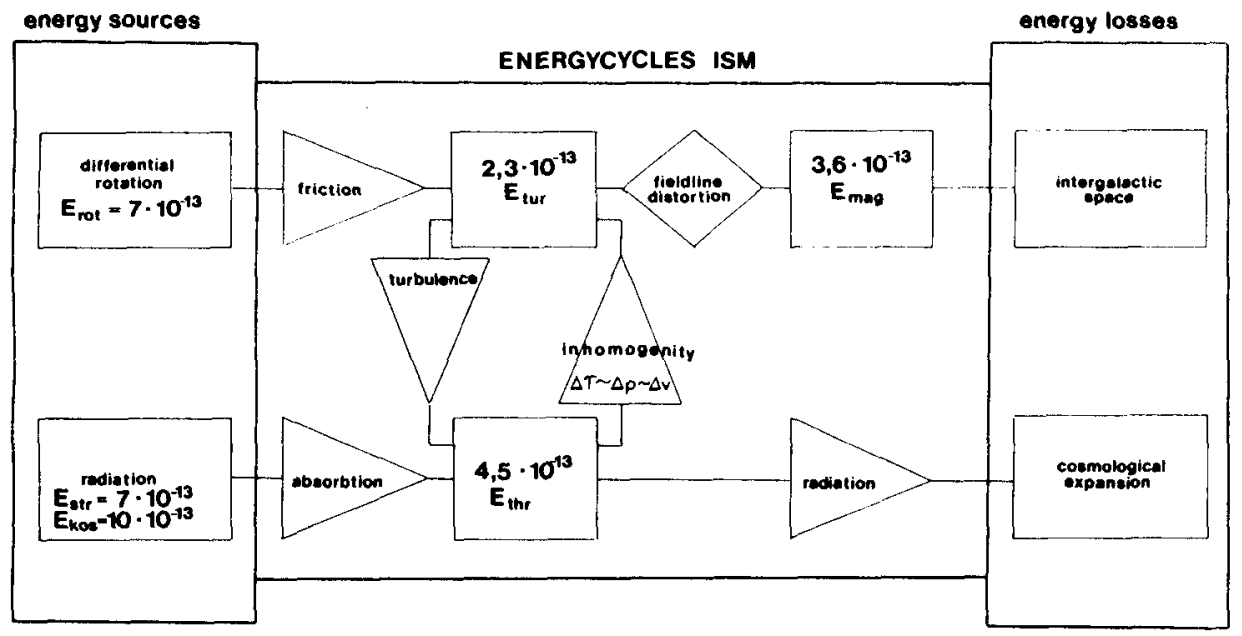

Fig. 1: A flow diagram of the energy equilibrium in the interstellar medium is presented. The energy sources and the energy losses are linked with different reservoirs and dissipation processes. This visualizes the galactic ecosystem. The mean energies are given in erg $/ \mathrm{cm}^{3} ; E_{\text {rot }}=$ rotation, $E_{s t r}=$ radiation, $E_{k o s}=$ cosmic radiation, $E_{t u r}=$ turbulence, $E_{m a g}=$ magnetic, and $E_{t h r}=$ thermal . 


\section{Discussion}

J. Percy: In textbooks and other general astronomy books, there are several advantages to using photographs taken by amateurs:

i) They are often of very high quality.

ii) They are often of "everyday" objects that are discussed in school textbooks.

iii) Amateurs are usually pleased to contribute their work for publication.

iv) Readers can "identify" with photographs taken by people like themselves.

\section{TAKE THE "A" TRAIN" TO THE STARS}

John Pazmino and Sidney Scheuer

Amateur Astronomers Association, 1010 Park Avenue, New York, NY 10028, U.S.A.

Astronomers, in addition to their scholarly and academic functions, have the mission to bring enlightenment to the people. In the City of New York, astronomers fulfill this mission through the Amateur Astronomers Association. Over the decades, the Association, or AAA, evolved a multi-faceted scheme of public enlightenment in astronomy. Under this scheme, astronomy in New York City has become a freestanding cultural amenity on a par with streetfairs, artshows, plays, and parades.

Once a month during the school year, the Association presents a formal public lecture on astronomy. These are convened in the American Museum of Natural History, the ancestral birthplace of the AAA. Occasionally, lectures are featured at a large university in the City for time and place variety. At these lectures, a professional astronomer explains some contemporary topic on a first-year college level, illustrated by slides and viewgraphs. The lectures - and all public activities of the AAA - are free of any charge. Area high schools and colleges employ the AAA lectures as an extra-curricular activity for their students.

In the summer, the AAA stages public stargazing in Carl Schurz Park, along the East River in Manhattan. Though located in the dense Upper East Side, Carl Schurz Park offers clear views of about two-thirds of the sky with adequate shielding from nearby lights. These sessions, convened monthly in clear weather, feature the celestial sights of the season: the moon, planets, clusters and nebulae, and double stars. Telescopes and charts are provided by the AAA.

The Amateur Astronomers Association operates the astronomy program at Gateway National Recreation Area under contract with the U.S. National Park Ser-

${ }^{2} E d$. Note: The "A" train is one of the New York subway routes that serves Harlem; it was made especially famous by Duke Ellington's jazz piece "Take the "A" Train." The authors can provide detailed information about the " $\mathrm{A}$ " train for any readers who have a special interest in this topic. 